\title{
Receding Horizon Control of Hybrid Linear Delayed Systems: Application to Sewer Networks
}

\author{
Bernat Joseph-Duran, Carlos Ocampo-Martinez and Gabriela Cembrano
}

\begin{abstract}
A control-oriented hybrid linear model for water transport in sewer networks is proposed as a suitable framework for the computation of real-time controllers for the minimization of flooding in presence of heavy-rain events. The model is based on individual network elements (sewers, gates, weirs and tanks) and does not rely on topological simplifications, thus providing a better description of the hydrological and hydraulic phenomena than in similar works. Using a generic form of a hybrid linear model, a simple matrix-based procedure for the formulation and solution of an optimal control problem is also presented. This procedure is applied to the sewer network model for the case study of a part of the Barcelona sewer network in a receding horizon control strategy, showing the effectiveness of the proposed control approach.
\end{abstract}

\section{INTRODUCTION}

Combined sewer networks carry both wastewater and storm-water together. During low to moderate rain events, this water is carried to wastewater treatment plants, where it is treated before being released to the receiving environment. However, during heavy-rain events the network capacity can be easily overloaded, causing urban surface flooding as well as untreated water discharges to the environment, known as combined sewer overflows (CSO), to happen.

Cities with combined sewer networks are usually equipped with detention tanks at some strategic points to store stormwater at the peak rain intensity periods and later release it at lower flow rates. The management of these detention tanks as well as other water distribution elements such as redirection gates plays a central role in avoiding flooding and maximizing water treatment.

Due to the uncertain distribution of the rain inflows to the network, global real-time control through network monitoring and rain intensity forecasts is regarded as the best control option, c.f. [1]. Physical models describing the transport of water in open channels involve the solution of partial differential equations, which makes them unsuitable for real-time control purposes due to high computational burden. Therefore, simple mathematical models are needed to simulate the network behavior to compute the best control actions at each time step.

The main contribution of this work, consists in a sewer network model that improves the hydraulic and hydrologic description of the network of previous works [2], [3], [4], [5], [6], [7], while still being computationally fast enough to be used in an optimization-based real-time control approach. The model describes the flows and volumes in each

All authors are with the Institut de Robòtica i Informàtica Industria (CSIC-UPC), Carrer Llorens i Artigas, 4-6, 2a planta 08028 Barcelona (Spain). bjoseph@iri.upc.edu network element, making it closer to the physical model than conceptual approaches based on modeling network areas as virtual tanks [2], [4], [5]. Other specific novelties with respect to similar models include overflow, weir flow and flooding volume descriptions. This improved modeling allows for better accuracy in the predictions as well as precise quantification of the management objectives, thus becoming a better option for any model-based control strategy than the previously mentioned ones.

From the particular network model, a general expression for a hybrid linear delayed model has been obtained and a simple procedure for the formulation of an optimal control problem (OCP) has been developed. The OCP has been formulated and solved for the model in the case study of a part of the Barcelona sewer network in a receding horizon control strategy.

The paper is organized as follows: in Section II a general expression for a hybrid linear delayed model and some considerations on the model evaluation procedure are provided. Section III is devoted to the sewer network modeling approach, together with some details about its hybrid linear delayed formulation. In Section IV the procedure to formulate the optimal control problem from a general hybrid linear delayed model is developed. Section V describes the particular case study on which the model has been applied together with some validation results. The improvement of the presented receding horizon control approach against passive control is presented in Section VI. Finally, conclusions of the study are given in Section VII together with some future research lines.

\section{HYBRID LINEAR DELAYED SYSTEMS}

Hybrid systems allow modeling systems involving both continuous and binary variables. These systems are defined not only by a set of dynamic equations, but also by a set of inequalities. If the system is properly defined, these inequalities define uniquely the values of the binary variables that are also involved in the dynamic equations [8].

In a delayed system setting both dynamic equations and inequalities involve the system variables at different time steps. In the discrete-time linear case the system reads

$$
\begin{aligned}
& \sum_{i=0}^{T} M_{i} X(t-i)=m(t), \\
& \sum_{i=0}^{T} N_{i} X(t-i) \leq n(t),
\end{aligned}
$$


where $t \in \mathbb{Z}$ is the discrete time variable and

$$
X(t-i)=\left(x_{1}(t-i), \ldots, x_{n}(t-i)\right)^{\top}, i=0, \ldots, T,
$$

with $x_{j}(t-i) \in \mathbb{R}$ for a subset of indices $j \in \mathcal{C} \subset$ $\{1, \ldots, n\}$ and $x_{j}(t-i) \in\{0,1\}$ for a subset of indices $j \in \mathcal{B} \subset\{1, \ldots, n\}$. Index sets $\mathcal{C}$ and $\mathcal{B}$ are such that $\mathcal{C} \cap \mathcal{B}=\varnothing$ and $\mathcal{C} \cup \mathcal{B}=\{1, \ldots, n\} . M_{i}, i=0, \ldots, T$, and $N_{i}, i=0, \ldots, T$, where $T$ is the maximum system delay, are matrices of appropiate dimensions. Discrete variables can have a direct physical meaning but more commonly arise in the formulation of piecewise equations of the model.

Vectors $X(t-i), i=0, \ldots, T$, include all system variables, making no distinction whether they are either state variables or controlled variables. The influence of any disturbance variable at any time step is included in vectors $m(t)$ and $n(t)$.

Given $X(t-i), i \geq 1$, system (1) is to be solved for $X(t)$ by means of a constraint satisfaction problem (CSP, [9]) when controlled variables are assigned a fixed value (to use the model for simulation purposes) or by means of an optimization problem if controlled variables are set free to minimize a performance criterion. As will be explained in Section IV, system (1) can be extended to solve, not only for $X(t)$, but also for $X(t+1), \ldots, X(t+H)$, for a given future horizon $H$.

\section{SEWER NETWORK MODEL}

\section{A. Model Overview}

A hybrid linear delayed model has been chosen as the modeling framework for the sewer network description since it efficiently deals with three main aspects of the problem. Firstly, the presence of delays in the model is a common element in any water transportation model. Secondly, the hybrid approach allows to model the presence of overflows in the network, which only occur when a given flow is above a threshold value, thus according to a logical condition. Finally, the linear framework is specially suited since sewer networks usually have a high number of variables, making the systems belong to the large-scale class.

The model describes the flows through sewers, gates and weirs and volumes stored in tanks in a discrete time setting. The main equations account for delay and wave attenuation in sewers and mass balance in junctions.

The overflows causing urban flooding and the flows over weirs are described by means of minimum and maximum functions, which can be easily formulated in the hybrid framework of the model.

A novel feature of the model consists in keeping track of the overflow volume by means of a virtual tank. This volume is stored in the tank until the overflow has finished and then is returned to the network as an inflow to the junction where the overflow has occurred. Variables involved in the model are collected in Table I.

\section{B. Mixed Logical Dynamical Systems Approach}

The Mixed Logical Dynamical (MLD) systems is a framework for modeling and control of systems governed by linear
TABLE I

SYSTEM VARIABLE NOTATION

\begin{tabular}{lccc} 
Description & Symbol & Units & Indexing \\
\hline \hline Flow entering sewers & $q_{i}^{\text {in }}(t)$ & $\mathrm{m}^{3} / \mathrm{s}$ & $i=1 \ldots n_{q}$ \\
\hline Flow leaving sewers & $q_{i}^{\text {out }}(t)$ & $\mathrm{m}^{3} / \mathrm{s}$ & $i=1 \ldots n_{q}$ \\
\hline Volume in tanks & $v_{i}(t)$ & $\mathrm{m}^{3}$ & $i=1 \ldots n_{v}$ \\
\hline Flow under gates & $g_{i}(t)$ & $\mathrm{m}^{3} / \mathrm{s}$ & $i=1 \ldots n_{g}$ \\
\hline Flow over weirs & $w_{i}(t)$ & $\mathrm{m}^{3} / \mathrm{s}$ & $i=1 \ldots n_{w}$ \\
\hline Overflows & $f_{i}(t)$ & $\mathrm{m}^{3} / \mathrm{s}$ & $i=1 \ldots n_{f}$ \\
\hline Flood runoff flow & $q_{t_{i}}(t)$ & $\mathrm{m}^{3} / \mathrm{s}$ & $i=1 \ldots n_{f}$ \\
\hline Overflow volume & $v_{t_{i}}(t)$ & $\mathrm{m}^{3} / \mathrm{s}$ & $i=1 \ldots n_{f}$ \\
\hline Rain inflow & $c_{i}(t)$ & $\mathrm{m}^{3} / \mathrm{s}$ & $i=1 \ldots n_{c}$ \\
\hline
\end{tabular}

dynamics together with switching behaviors arising from logical statements involving the variables of the system [8].

To this end, binary variables describing the truth value of the fulfillment of linear inequalities are defined. Using these variables, any statement constructed using the usual logical operators ('and', 'or', negation and implication) concerning the truth value of linear inequalities can be reformulated as further equalities and inequalities.

Thus, the MLD framework allows to model any system behavior consisting in switching between different linear dynamics depending on the different combinations of the truth value of a set of linear inequalities. MLD systems have been shown to be equivalent to other system modeling formats including linear complementarity systems, extended linear complementarity systems, piecewise affine systems, and max-min-plus-scaling systems [10].

Further details on the particular procedure to reformulate the model equations in the MLD from and to obtain the final system expression (1) can be found in [11].

The values of all the parameters involved in the model equations are obtained from simulation data as the ones that minimize the model error, as detailed in Section V.

\section{Model equations}

1) Mass balance equations: For each sewer $i=1 \ldots n_{q}$, the flow upstream is computed as the sum of the inflows at the junction where sewer is connected

$$
\begin{aligned}
q_{i}^{\text {in }}(t)= & \sum_{j=1}^{n_{q}} a_{i j}^{q} q_{j}^{\text {out }}(t)+\sum_{j=1}^{n_{w}} a_{i j}^{w} w_{j}(t)+\sum_{j=1}^{n_{f}} a_{i j}^{f} f_{j}(t)+ \\
& \sum_{j=1}^{n_{c}} a_{i j}^{q_{t}} q_{t_{j}}(t)+\sum_{j=1}^{n_{g}} a_{i j}^{g} g_{j}(t)+\sum_{j=1}^{n_{c}} a_{i j}^{c} c_{j}(t) .
\end{aligned}
$$

If $q_{i}$ is the only outflowing sewer connected to the junction, the nonzero $a_{i j}^{*}$ take the value 1 . Otherwise they take values in $(0,1)$ such that the sum of the coefficients of all the outgoing sewers add to 1 .

2) Flow equations: The flow downstream of each sewer is a convex combination of the upstream flows at two previous time steps. Hence, for each sewer $i=1 \ldots n_{q}$

$$
q_{i}^{\text {out }}(t)=a_{i} q_{i}^{\text {in }}\left(t-t_{i}\right)+\left(1-a_{i}\right) q_{i}^{i n}\left(t-t_{i}-1\right),
$$

with $a_{i} \in(0,1]$. This model has been chosen because the delay in sewers is not a multiple of the sampling time unless it is chosen very small, which would lead to a high 
number of variables in the problem to cover a reasonable prediction horizon. By means of a convex combination of two consecutive time steps, delays of any magnitude can be suitably approximated.

3) Weir model: Weirs are passive flow-regulation elements that redirect part of the inflow to a sewer to a secondary one, called a spillway, when a certain water level is reached [12]. Since the proposed model does not include water levels, an approximation in terms of flow is used. The flow over a weir attached to a junction with total inflow $z_{w}(t)$ is computed as

$$
w(t)=\max \left\{0, a_{w}\left(z_{w}(t)-q_{w}^{\max }\right)\right\},
$$

where $q_{w}^{\max }$ is the inflow value at which water starts flowing through the spillway. As mentioned before, the flow over a weir does not actually depend on flow values but on water level, thus it is observed in data obtained from a complete physical model simulator that flow values at the main sewer can reach values greater than $q_{w}^{\max }$. This fact is suitably approximated by the introduction of parameter $a_{w} \in(0,1)$.

4) Overflow model: Overflows are defined in junctions in a way completely analogous to the weir flow:

$$
f(t)=\max \left\{0, a_{f}\left(z_{f}(t)-q_{f}^{\max }\right)\right\},
$$

where $z_{f}(t)$ is the total inflow to the junction and $q_{w}^{\max }$ is the inflow value at which overflow starts. Again, $a_{f} \in(0,1)$ is introduced to better approximate the fact that the outflow can be higher than $q_{f}^{\max }$.

Although overflows could be defined in every network junction, it is better to define them only at those prone to suffer from overflow events. This junctions can be easily determined from simulation data. Avoiding the definition of overflow variables at those junctions where overflows are very unlikely to occur improves the model computational speed since it is strongly dependent on the amount of binary variables, specially in the optimal control case.

5) Flood runoff model: A novel feature of the proposed model consists in keeping track of the volume that goes out of the network through overflows to let it return to the network when the overflow event has finished. To this end, the overflow in a junction $f(t)$ is stored in a virtual tank with volume $v_{t}(t)$ using

$$
v_{t}(t)=v_{t}(t-1)+\Delta t\left(f(t-1)-q_{t}(t-1)\right),
$$

with $q_{t}(t)$, the emptying flow defined as follows:

$$
q_{t}(t)=\min \left\{\max \left\{0, b_{f}\left(q_{f}^{\max }-z_{f}(t)\right)\right\}, \frac{v_{t}(t)}{\Delta t}\right\} .
$$

The tank can never provide more flow than that which would empty it in a single time step (i.e. $v_{t}(t) / \Delta t$ ), thus the minimum is applied. If there is enough volume available, the tank empties at a rate $b_{f}$ proportional to the difference between the overflow threshold $q_{f}^{\max }$ and the inflow $z_{f}(t)$, provided this difference is a positive number. If $b_{f}=1$ the tank empties as fast as possible making the outgoing sewer at the junction have a flow value $q_{f}^{\max }$. Other values $0<b_{f}<1$ let the tank empty at slower rates to better fit simulation data.

\section{THE OPTIMAL CONTROL PROBLEM}

The Optimal Control Problem (OCP) associated with system (1) consists in determining which values for the controlled variables minimize an objective function that quantifies performance of the system according to its dynamics. It is assumed that reliable predictions for the disturbance variables of the system are available at times $t, \ldots, t+H$.

For future time steps, system (1) reads

$$
\begin{gathered}
\sum_{i=0}^{T} M_{i} X(t-i+k)=m(t+k), \\
\sum_{i=0}^{T} N_{i} X(t-i+k) \leq n(t+k) .
\end{gathered}
$$

Thus, the dynamics of the system for $X(t), \ldots, X(t+H)$, are described by coupling the above system for $k=0 \ldots H$. The resulting system can be written in compact form as

$$
\begin{gathered}
\mathcal{M}_{1} \mathcal{X}(t)=\mathcal{M}_{2} \mathcal{X}_{0}(t)+\mathcal{M}_{3}(t), \\
\mathcal{N}_{1} \mathcal{X}(t) \leq \mathcal{N}_{2} \mathcal{X}_{0}(t)+\mathcal{N}_{3}(t),
\end{gathered}
$$

with

$$
\begin{gathered}
\mathcal{X}(t)=\left(X(t+H)^{\top}, \ldots, X(t)^{\top}\right)^{\top}, \\
\mathcal{X}_{0}(t)=\left(X(t-1)^{\top}, \ldots, X(t-T)^{\top}\right)^{\top},
\end{gathered}
$$

and

$$
\begin{aligned}
& \mathcal{M}_{1}=\left(\begin{array}{ccccccc}
M_{0} & M_{1} & \ldots & \ldots & M_{T} & & \\
& \ddots & & & & \ddots & \\
& & M_{0} & M_{1} & \ldots & \ldots & M_{T} \\
& & & M_{0} & \ldots & \ldots & M_{T-1} \\
& & & & \ddots & & \vdots \\
& & & & & M_{0} & M_{1} \\
& & & & & & M_{0}
\end{array}\right) H+1 \text { blocks } \\
& \left.\mathcal{M}_{2}=-\left(\begin{array}{ccccc} 
& & & \\
M_{T} & & & \\
M_{T-1} & M_{T} & & \\
\vdots & \vdots & \ddots & \\
M_{2} & M_{3} & \ldots & M_{T} \\
M_{1} & M_{2} & \ldots & M_{T-1} & M_{T}
\end{array}\right)\right\} H+1 \text { blocks } \\
& \mathcal{M}_{3}(t)=\left(m(t+H)^{\top}, \ldots, m(t)^{\top}\right)^{\top},
\end{aligned}
$$

and analogous expressions for $\mathcal{N}_{1}, \mathcal{N}_{2}$ and $\mathcal{N}_{3}$. For the particular shape of these matrices it has been assumed that $H>T$, that is, the prediction horizon is bigger than the largest delay in the system. Although this condition is not necessary to formulate the OCP, it is a common and reasonable assumption since it allows to take into account the complete system dynamics in the predictions implicitly made by the optimizer.

Finally, the OCP at time step $t$ is formulated by adding all dynamics and MLD inequalities of the system as constraints of an optimization problem of the form:

$$
\begin{aligned}
\min _{\mathcal{X}(t)} J(\mathcal{X}(t)) & =c^{\top} \mathcal{X}(t) \\
\text { s.t. } \mathcal{M}_{1} \mathcal{X}(t) & =\mathcal{M}_{2} \mathcal{X}_{0}(t)+\mathcal{M}_{3}(t), \\
\mathcal{N}_{1} \mathcal{X}(t) & \leq \mathcal{N}_{2} \mathcal{X}_{0}(t)+\mathcal{N}_{3}(t), \\
A_{\text {eq }} \mathcal{X}(t) & =b_{\text {eq }}(t) \\
A_{\text {ineq }} \mathcal{X}(t) & \leq b_{\text {ineq }}(t)
\end{aligned}
$$


In this case, a linear function has been used and the corresponding optimization problem becomes a Mixed Integer Linear Program (MILP). More generally, a quadratic function could be considered and the corresponding optimization problem would become a Mixed Integer Quadratic Program (MIQP). Both the MILP and the MIQP versions of the OCP can be solved by using specialized optimization software. Notice that the number of discrete variables depends on the number of overflow points and weirs in the network and these are only present in small quantities. Therefore, the resulting optimization problems can be solved in short computing times.

Notice, also, the addition of further equalities, $A_{e q} \mathcal{X}(t)=$ $b_{\text {eq }}(t)$, and inequalities, $A_{\text {ineq }} \mathcal{X}(t) \leq b_{\text {ineq }}(t)$. These are any other additional constraints to be added to the system to improve its behavior. Typical ones include bounds on the controlled variables due to physical limits and bounds on its variation rates, to ensure smooth control actions.

\section{CASE STUDY AND MODEL VALIDATION}

To validate the model and test the formulation approach for the OCP presented in the previous sections a part of the Barcelona sewer network has been studied. This network, spanning an area of approximately $26 \mathrm{~km}^{2}$, has proven especially representative of the overflow and CSO phenomena. Full information of the network is available thanks to the data provided by the company responsible of its management CLABSA (Clavegueram de Barcelona S.A.). The company has also provided a full implementation of the network in a physical model-based simulator of sewer networks (MOUSE, [13]). This implementation has been used to carry simulations to get the data necessary for model calibration and validation.

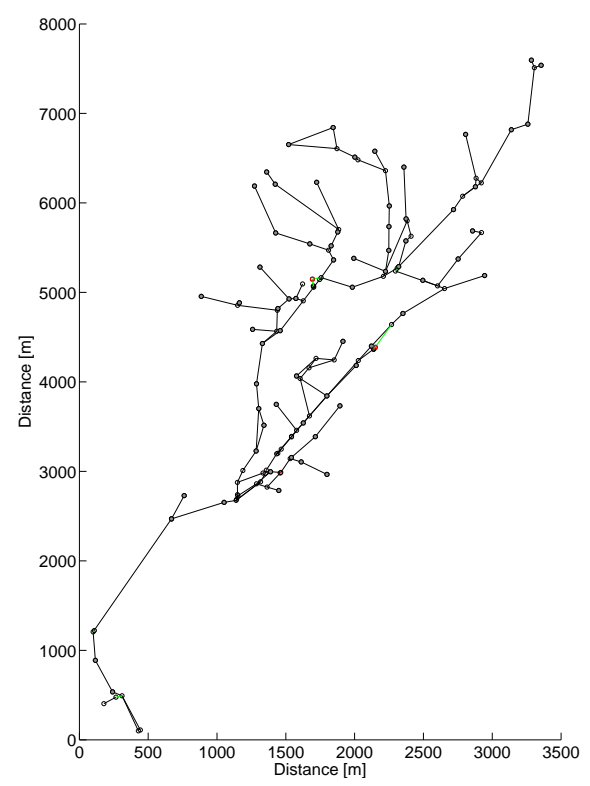

Fig. 1. Diagram of Riera Blanca sewer network after simplification.

After a mild network simplification consisting in considering junctions where more than two sewers meet (thus considering all the linear trams as single sewers) or junctions where rain inflow is defined, the network consists in 147 sewers regulated by the presence of 2 detention tanks, 3 weirs and 10 gates. After simulation using several rain events of different intensities, overflow has been considered in 26 junctions. A sample time of $\Delta t=1 \mathrm{~min}$ was chosen and a maximum delay of $T=9$ min was obtained from simulation data.

The network converges at its downstream end to a single big sewer with an in-line detention capacity of about $32000 \mathrm{~m}^{3}$, which has been modeled as a tank for optimal control purposes, controlled by a gate at its downstream end. The outflow of this sewer is directed to a Wastewater Treatment Plant (WWTP) with an inflow capacity of $2 \mathrm{~m}^{3} / \mathrm{s}$ and all the flow above this value is released to the sea as CSO.

\section{A. Rain Events}

Ten rain events of different durations and intensities have been used for parameter calibration: five real-rain events and five designed ones. After some tests and trial-and-error experiments, a final set of parameters has been determined as the one providing the best fits. The five real-rain scenarios have been used for validation.

Rain data is provided to the simulator as pluviometer data and is transformed to flow entering the network at some of the junctions by means of a dynamic model (Kinematic Wave Model B, [14]) that makes use of the physical characteristics of the runoff catchment associated to the junction.

\section{B. Model Validation}

To validate the model, the following expressions for the error have been used. For each sewer define $\bar{e}_{i}, i=1 \ldots n_{q}$, as the mean value of the accumulated absolute error over the simulation horizon $H$

$$
\bar{e}_{i}=\frac{1}{H} \sum_{t=1}^{H}\left|q_{i}^{i n}(t)-\hat{q}_{i}^{i n}(t)\right|,
$$

where $\hat{q}_{i}^{i n}(t)$ are the flow values computed by the simulator. To obtain a description of the overall network behavior, the mean and the maximum of these errors are used as indicators:

$$
E_{1}=\frac{1}{n_{q}} \sum_{i=1}^{n_{q}} \bar{e}_{i}, \quad E_{2}=\max _{i} \bar{e}_{i} .
$$

Table II shows the results obtained for the five real-rain events together with its duration $H$ in time steps.

TABLE II

MODEL ERROR

\begin{tabular}{cccc} 
Episode & $E_{1}$ & $E_{2}$ & $H$ \\
\hline \hline $26-08-2002$ & 0.082 & 1.115 & 383 \\
\hline $17-09-2002$ & 0.073 & 0.828 & 633 \\
\hline $09-10-2002$ & 0.112 & 1.023 & 864 \\
\hline $29-01-2006$ & 0.063 & 0.766 & 658 \\
\hline $15-08-2006$ & 0.090 & 1.073 & 539 \\
\hline \hline
\end{tabular}

As expected, the model accuracy deteriorates as the simulation time increases due to the cumulative effect of the 
error. This fact is shown in Table III for the two most intense episode, which will be later used to test the OCP approach.

TABLE III

MODEL ERROR FOR INCREASING SIMULATION TIMES

\begin{tabular}{c|cc|cc}
\multirow{2}{*}{$H$} & \multicolumn{2}{|c|}{$09-10-02$} & \multicolumn{2}{c}{$15-08-06$} \\
\cline { 2 - 5 } & $E_{1}$ & $E_{2}$ & $E_{1}$ & $E_{2}$ \\
\hline \hline 30 & 0.017 & 0.080 & 0.013 & 0.082 \\
\hline 60 & 0.042 & 0.531 & 0.035 & 0.216 \\
\hline 90 & 0.043 & 0.415 & 0.092 & 1.046 \\
\hline 120 & 0.105 & 1.317 & 0.104 & 1.193 \\
\hline \hline
\end{tabular}

It can also be seen that the error increases in the sewers of the downstream part of the network. This is mainly due to two reasons: first, the error from all the upstream sewers accumulates. Secondly, the sewers in this part are bigger and of lower slope and the nonlinear effects of open channel flow become more dominant, making the linear approximation less accurate. Figure 2 shows an example of this phenomenon, with flows as computed by MOUSE simulator and the proposed model.
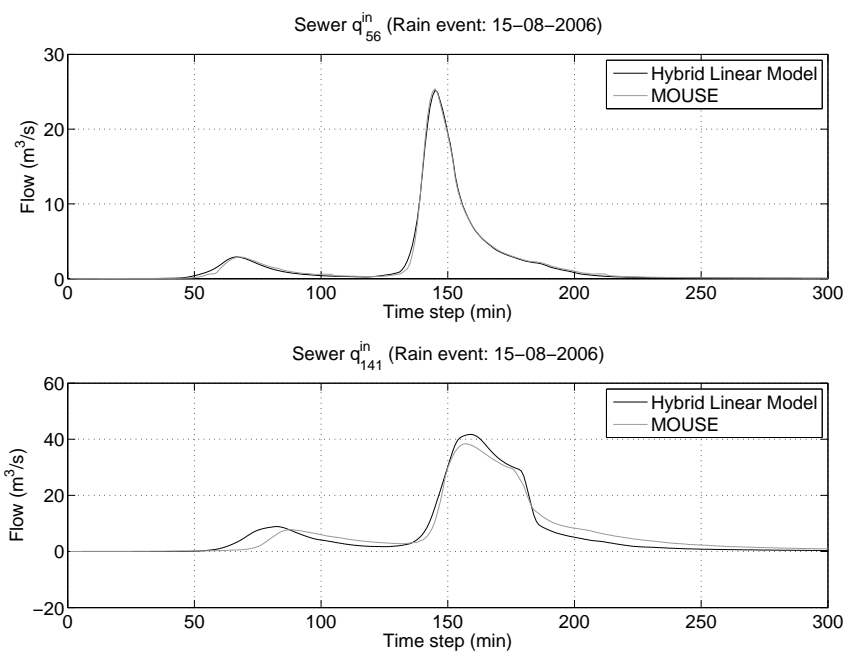

Fig. 2. Flow in sewers as computed by MOUSE simulator and the presented model for an upstream sewer $\left(q_{56}^{i n}\right)$ and a downstream one $\left(q_{141}^{i n}\right)$.

\section{RECEDING HORIZON CONTROL RESULTS}

The three main objectives of the network management consist in:

1) Avoid overflow in urban areas.

2) Avoid CSO discharges to the sea.

3) Maximize WWTP usage.

Each individual objective has been translated into its corresponding objective function for problem (2) as

$$
\begin{aligned}
J_{O F}(\mathcal{X}(t)) & =c_{O F}^{\top} \mathcal{X}(t), \\
J_{C S O}(\mathcal{X}(t)) & =c_{C S O}^{\top} \mathcal{X}(t), \\
J_{W W T P}(\mathcal{X}(t)) & =-c_{W W T P}^{\top} \mathcal{X}(t),
\end{aligned}
$$

by summing up the corresponding system variables. Notice that the WWTP volume is to be maximized, thus the negative sign. Besides this three single objective functions, the following two multi-objective ones have been considered:

$$
\begin{gathered}
J_{1}=J_{O F}+J_{C S O} \\
J_{2}=J_{O F}+J_{C S O}+10^{-1} J_{W W T P} .
\end{gathered}
$$

This multi-objective functions have been chosen to study whether the different management objectives compete among each other or, otherwise, cooperate to finally obtain a better overall network performance. The weights for each objective have been chosen by a trial-and-error procedure taking into account that, as a management decision, overflows and CSO discharges are regarded as more important than WWTP usage. Further tuning techniques regarding these weights are out of the scope of this paper.

The receding horizon control simulation of the network has been carried as follows: at time $t=1$ the optimal control problem is solved for a prediction horizon of $H=30 \mathrm{~min}$ (the estimated time for water to travel along the whole network). Since the optimal control problem solves for both the control actions and the network dynamics, the variables corresponding to the first five minutes of the event are kept, and the initial conditions $\mathcal{X}_{0}$ are updated to solve the problem again at time step $t=5$. The whole procedure is repeated solving the optimal control problem for a prediction horizon of $H=30$ every five minutes and keeping only the part of the solution corresponding to the first five minutes of the optimal result to build the overall solution.

The optimization problems corresponding to a prediction horizon of $H=30$ are composed of 5460 continuous variables and 690 binary variables together with 4830 equality constraints and 17160 inequality constraints. All problems have been solved using CPLEX ${ }^{\mathrm{TM}}$ v12.4 [15] MILP solver with standard settings, available thanks to IBM Academic Initiative [16].

The results of the receding horizon procedure applied to the two most intense real-rain episodes available together with the results obtained with the passive/no-control approach implemented in the simulator, consisting in keeping all gates at fixed positions, are presented in Table IV. Since the real control of the network is performed by expert operators on the basis of historical and simulation data, no

\begin{tabular}{|c|c|c|c|c|}
\hline Episode & Objective & $\begin{array}{c}\text { Overflow } \\
{\left[\times 10^{3} \mathrm{~m}^{3}\right]}\end{array}$ & $\begin{array}{c}\mathrm{CSO} \\
{\left[\times 10^{3} \mathrm{~m}^{3}\right]}\end{array}$ & $\begin{array}{c}\text { WWTP } \\
{\left[\times 10^{3} \mathrm{~m}^{3}\right]}\end{array}$ \\
\hline \multirow{6}{*}{$09-10-02$} & Overflow & $0.44(-97.4 \%)$ & $401.41(-19.6 \%)$ & $0.00(-100.0 \%)$ \\
\hline & $\mathrm{CSO}$ & 0. & & $169.03(+$ \\
\hline & WWTP & $0.44(-97.4 \%)$ & $400.83(-19$ & $144.40(+79.6 \%)$ \\
\hline & $J_{1}$ & $0.44(-$ & $286.06(-4)$ & 139.18 \\
\hline & $J_{2}$ & $0.44(-$ & 283.02 & 190.88 \\
\hline & No Control & 16.76 & 499.15 & 80.41 \\
\hline \multirow{6}{*}{$15-08-06$} & Overflow & $0.29(-94.2 \%)$ & $72.90(-18.8 \%)$ & $0.00(-100.0 \%)$ \\
\hline & & 0.29 & 6.11( & 105.44 \\
\hline & WWTP & $0.29(-94$ & $61.50(-31$ & 55.22 \\
\hline & $J_{1}$ & $0.29(-94$ & $6.02(-93.3 \%)$ & 46.82( \\
\hline & $J_{2}$ & $0.29(-94.2 \%)$ & $4.39(-95.1 \%)$ & $112.34(+214.7 \%)$ \\
\hline & No Control & 5.06 & 89.82 & 35.70 \\
\hline
\end{tabular}
results from other global control strategies are available for comparison.

TABLE IV

RECEDING HORIZON RESULTS 
Several interesting features of the receding horizon strategy can be stated from these results. First, it is worth noticing that the proposed receding horizon strategy, although based in solving optimal control problems, does not provide an optimal performance along the entire simulation horizon. Since the decisions are made at each time step based on rain inflow information of a limited time window, it can happen that minimization of a single objective provides worse results than the minimization of another objective function and, in fact, this is the case for WWTP maximization. This is because, the optimal WWTP usage for the short horizon OCP is achieved by means of high flow rates at the downstream end of the network. Due to the limitation of $2 \mathrm{~m}^{3} / \mathrm{s}$ at the WWTP, most of this flow is directed to the sea as CSO, thus leaving the network and not being available for treatment at future time steps. On the other hand, single or combined minimization of the CSO objective takes advantage of WWTP usage while retaining the water in the network for future treatment, providing better results for both CSO minimization and WWTP maximization.

Secondly, notice that overflows are always minimized, no matter which objective function is used. Overflows occur in the middle part of the network, where flows from upstream parts converge, but sewers are not as big as in the downstream end. The proper management of the redirection gates and detention tanks helps achieving lower flow values at this part of the network, reducing or avoiding overflows. As a consequence, flow values at the downstream end are also lower, which contributes avoiding CSO discharges and maximize WWTP usage. Thus, overflow minimization is always beneficial for the minimization of the other objectives. This fact explains why combining the minimization of overflow and CSO objectives in the multi-objective function $J_{1}$ provides almost the same results as minimizing each one individually: the two objectives are not in conflict.

Finally, it can be seen that objective function $J_{2}$ provides the best overall results. Thus, it can be stated that the three objectives, when used together, contribute to an overall performance improvement. According to the previous discussions, objective function $J_{2}$ takes advantage of the benefits of the combination of the CSO and WWTP objectives while still obtaining minimal overflow values. Moreover, the presence of the WWTP term in the objective function forces the tanks to empty after the rain has ceased so that the volume stored in them can be treated, which is not the case for multiobjective function $J_{1}$. This is a very important feature since it helps finishing the rain event with empty tanks, which is very desirable in case rain starts again.

\section{CONCLUSIONS AND FUTURE WORK}

This paper has proposed a control-oriented modeling framework suitable for the design and implementation of optimization-based real-time controllers. The presented model, optimal control problem and receding horizon approach conform the preliminary steps towards a model predictive control problem formulation. The model provides a proper description of the network dynamics, since flows at any sewer are computed, and can be applied to any network for which detailed physical data is available. It has proven to provide a good trade-off between precision and computation speed, thus becoming a suitable model for a predictive control approach. The optimal control problem can be easily implemented using the matrices of the system and the resulting OCP can be solved in real time.

Future work includes a closed-loop real-time implementation against a physical model-based simulator using on-line calibration techniques, model adaptation to the intensity of the rain scenarios to better approximate the sewer delays and tuning of the objective functions. Another important research line is to develop more accurate models for big sewers with low slope controlled by a gate at its downstream end, since this kind of sewers show strong nonlinear features, which are not properly approximated by linear models.

\section{ACKNOWLEDGMENTS}

This work has been partially funded by the Spanish project WATMAN (DPI2009-13744), the EU Project EFFINET (FP7-ICT-2011-8-31855), and the JAE-PreDoc program of the Spanish National Research Council (CSIC). The authors specially thank the invaluable collaboration of CLABSA staff during the preparation of this paper.

\section{REFERENCES}

[1] M. Schütze, A. Campisano, H. Colas, W. Schilling, and P. Vanrolleghem, "Real time control of urban wastewater systems - where do we stand today?," Journal of Hydrology, vol. 299, no. 3-4, pp. 335348, 2004.

[2] M. Gelormino and N. Ricker, "Model-predictive control of a combined sewer system," Int. J. Control, vol. 59, no. 3, pp. 793-816, 1994.

[3] M. Marinaki and M. Papageorgiou, Optimal Real-time Control of Sewer Networks. London: Springer, 2005.

[4] G. Cembrano, J. Quevedo, M. Salamero, V. Puig, J. Figueras, and J. Martí, "Optimal control of urban drainage systems. A case study," Control Engineering Practice, vol. 12, no. 1, pp. 1-9, 2004.

[5] V. Puig, G. Cembrano, J. Romera, J. Quevedo, B. Aznar, G. Ramón, and J. Cabot, "Predictive optimal control of sewer networks using CORAL tool: application to Riera Blanca catchment in Barcelona," Water Science and Technology, vol. 60, no. 4, pp. 869-878, 2009.

[6] C. Ocampo-Martínez, V. Puig, G. Cembrano, and J. Quevedo, "Application of predictive control strategies to the management of complex networks in the urban water cycle," IEEE Control Systems, vol. 33, no. 1 , pp. 15-41, 2013.

[7] C. Ocampo-Martínez, Model predictive control of wastewater systems. Advances in industrial control, London: Springer, 2011.

[8] A. Bemporad and M. Morari, "Control of systems integrating logic, dynamics, and constraints," Automatica, vol. 35, no. 3, pp. 407-427, 1999.

[9] L. Jaulin, M. Kieffer, O. Didrit, and E. Walter, Applied Interval Analysis. Computational Intelligence and Complexity, London: SpringerVerlag, 2001.

[10] W. P. M. H. Heemels, B. De Schutter, and A. Bemporad, "Equivalence of hybrid dynamical models," Automatica, vol. 37, pp. 1085-1091, July 2001.

[11] B. Joseph-Duran, C. Ocampo-Martinez, and G. Cembrano, "Hybrid linear sewer network modeling," Tech. Rep. IRI-TR-09-07, Institut de Robòtica i Informàtica Industrial, CSIC-UPC, 2013.

[12] X. Litrico and V. Fromion, Modelling and Control of Hydrosystems. London: Springer, 2009.

[13] DHI Software, MOUSE User Guide, 2007.

[14] DHI Software, MOUSE Surface Runoff Models, 2007.

[15] CPLEX ${ }^{\mathrm{TM}}$, version 12.4 (2011). Sunnyvale, California: IBM ILOG, 2011.

[16] IBM ILOG, "IBM Academic Initiative." http: / / www.ibm.com/ academicinitiative, 2013. 